J. Math. Soc. Japan Vol. 13, No. 3, 1961

\title{
Axioms of infinity of set theory
}

\author{
By Gaisi TAKeut I \\ (Received March 7, 1960) \\ (Received June 7, 1960)
}

Mahlo, in his penetrating papers [5], [6], proposed an axiom of set theory, assuring, roughly speaking, the existence of sets with very large cardinals. Recently Lévy [3], [4] gave an elegant equivalent axiom, which he called the axiom of strong infinity. Mahlo's axiom can be also stated as follows in making use of the concept of the super-complete model introduced by Shepherdson [8]. (We remind that a set $a$ is called super-complete if and only if

$$
\forall x \forall y(x \in a \wedge(y \leqq x \vee y \in x) \vdash y \in a) ;
$$

and that a set $a$ is said to be a model of a set theory $T$, if and only if the theory holds, when all quantifiers of $T$ are restricted on $a$.)

Let $f$ be a function from sets to sets. A set $a$ will be called a fixed point of $f$, if and only if

$$
\forall x(x \in a \vdash f(x) \in a) .
$$

A class $A$ will be called dense, if and only if for every function there exists a fixed point of this function which is an element of $A$. Then Mahlo's axiom means: The class of all the super-complete models of Bernays-Gödel's set theory is dense.

To go further, let us denote with $M^{0}$ the Bernay-Gödel's set theory. In adding Mahlo's axiom to $M^{0}$, we obtain a new set theory $M^{1}$, with 'much more set' than in $M^{0}$. More generally, a stronger set theory $M^{i+1}$ is obtained in adding the axiom: The class of all the super-complete models of $M^{i}$ is dense, to $M^{i}, i=0,1,2, \cdots$. These $M^{i}$ will be called Mahlo's set theories.

Let $\widetilde{\mathfrak{R}}(B)$ mean that $B$ is the class of all the super-complete models of a Mahlo's set theory. $\mathscr{\Re}(A)$ (for a class $A$ ) will mean

$$
\exists B \exists x(\widetilde{\Re}(B) \wedge \forall a(a \in B \wedge x \subseteq a \wedge x \in a \vdash a \in A)) .
$$

Then we have easily

This work was done under Appointment supported by the International Cooperation Administration under the Visiting Research Scientists Program administered by the National Academy of Sciences of the United States of America. 
1. $\Re(0)$.

2. $\mathscr{\Re}\left(G_{0}\right)$,

where $G_{0}$ is the class of all the super-complete models of BernaysGödel's set theory.

3. $\forall A \forall B(\Re(A) \wedge A \subseteq B \vdash \mathscr{\Omega}(B))$.

4. $\forall A \forall x(\Re(A) \vdash \mathscr{\Re}((K a)(a \in A \wedge x \subseteq a \wedge x \in a)))$, where $(K a) \mathfrak{H}(a)$ means the class of sets satisfying $\mathfrak{P}(a)$, i. e. $\{a \mid \mathfrak{A}(a)\}$ in the usual notation.

5. $\forall A(\Re(A) \vdash$ ' $A$ is dense').

6. If a closed formula $\mathfrak{A}$ without $\mathscr{R}$ or $\widetilde{\mathfrak{R}}$ is provable in this system, then the class of all the super-complete models of $\mathfrak{A}$ satisfies $\mathscr{R}$.

Now we can make correspond to each function $f$ from sets to sets, a function $F$ from sets to classes defined by

$$
F(x)=(K b)(f(x) \in b) .
$$

A set $a$ is then a fixed point of $f$, if and only if

$$
\forall x(x \in a \vdash a \in F(x)) .
$$

In the following a set $a$ will be called a fixed point of a function $F$ from sets to classes, if and only if (*) holds. Using this notion, 5 can be strengthened into the following

$5^{\prime}$. Let $F$ be a function from sets to classes such that $\forall x(\mathscr{N}(F(x))$, and $B$ the class of all the fixed points of $F$. Then we have $\mathscr{I}(B)$. Symbolically :

$$
\forall A\left(\forall x \Re\left(A^{\prime \prime}\{x\}\right) \vdash \mathscr{N}\left((K a) \forall x\left(x \in a \vdash a \in A^{\prime \prime}\{x\}\right)\right)\right),
$$

where $A^{\prime \prime}\{x\}$ means $F(x)$ (cf. [1] ).

In adding a new predicate $\Re$ satisfying $1,2,3,4,5$, 6 to Bernays-Gödel's set theory, we obtain a theory $T^{0}(\Re)$, which is stronger than any Mahlo's theory. We can now strengthen this in doing as follows.

Let $a$ be a super-complete set and $k$ a set of subsets of $a$. The pair $\langle k a\rangle$ is called a super-complete model of $T^{0}(\Re)$, if and only if the theory holds when every $\mathscr{N}(*)$ in $T^{0}(\mathscr{R})$ is replaced by $* \in k$ and all quantifiers in $T^{0}(\Re)$ are restricted on $a$. The "Mahlo's axiom for $T^{0}(\Re)$ " will mean :

$(K a) \exists k(\langle k a\rangle$ is a super-complete model of $T(\Re))$ is dense.

In adding this axiom to $T^{0}(\Re)$, we obtain $T^{1}(\Re)$ and in repeating this, $T^{i}(\mathscr{R})$, $i=1,2, \cdots$. Thus a new predicate $\mathbb{S}_{1}$, and a set theory $T^{\circ}\left(\Re, \mathbb{S}_{1}\right)$ will be obtained from $T^{0}(\Re)$, just as we have obtained $\Re$ and $T^{0}(\Re)$ from $M^{0}$. In $T^{0}\left(\Re, \mathbb{S}_{1}\right)$ we have axioms corresponding to axioms $1-6,5^{\prime}$ in $T^{0}(\mathscr{R})$. In particular $5^{\prime}$ becomes

$$
\forall A\left(\forall x \mathbb{S}_{1}\left(A^{\prime \prime}\{x\}\right) \vdash \mathbb{S}_{1}\left((K\langle b a\rangle) \forall x\left(x \in a \vdash a \in \mathfrak{D}\left(A^{\prime \prime}\{x\}\right)\right)\right)\right),
$$


where $\mathfrak{D}$ means "domain" (cf. [1] ).

Beginning again from $T^{0}\left(\Re, \mathbb{S}_{1}\right)$ we can define $T^{i}\left(\mathscr{R}, \mathbb{S}_{1}\right) i=1,2, \cdots$, a new predicate $\mathbb{S}_{2}$, a new set theory $T^{0}\left(\Re, \mathbb{S}_{1}, \mathbb{G}_{2}\right)$ and so on.

In the next $\S 1$, we shall give a system of axioms of a set theory, which is stronger than all these theories $T^{i}\left(\Re_{1} \mathbb{S}_{1}, \mathbb{S}_{2}, \cdots\right)$. In $\S 2$ we give some properties of this system. It will be shown that our system contains 'infinite induction' as an inference sheme. It has a role of an axiom of infinity, because it assures the existence of the super-complete model of the system without the infinite induction. In $\S 3$ we discuss the questions about relative consistency of this system with a certain weaker system. We have not treated the problem of relative consistency of Gödel's $V=L$ with our system, because this seems to be very difficult. Finally we give in $\S 4$ still another system of axioms of the same nature.

It seems to the author that our system cannot be strengthened any more without introducing metamathematical concepts like the Gödel number into the system. He is not quite sure of the consistency of the system.

He is grateful to Prof. Gödel and Lévy for their kind discussions and criticisms during his stay in the United States.

\section{$\S 1$. Axioms and inferences.}

We use the notations and notions in [1]. Other than basic symbols in [1] we use basic symbols $K$ and $\$$.

The axioms fall into six groups. Groups $\mathrm{A}, \mathrm{D}$ and $\mathrm{E}$ are the same as in [1]. Instead of Groups $B$ and $C$ we use the following Groups $B^{\prime}$ and $C^{\prime}$.

Group B.$\quad \forall X_{1} \cdots \forall X_{m} \forall x_{1} \cdots \forall x_{n} \forall x(x \in K(u) \mathfrak{Y}(u) \mapsto \mathfrak{A}(x))$.

We shall consider the notations $E, \cap,-, \mathfrak{D}, \mathfrak{B},\left(\mathfrak{N} \mathfrak{n},\left(\mathfrak{S n v}_{2},\left(\mathfrak{S n v}_{3}\right.\right.\right.$ as the adequate abbreviations expressed by basic symbols. (We use $\cap$ instead of $\bullet$ in [1] , because we have to use $\cdot$ as the multiplication of integer. $\mathfrak{V}(A)$ expresses $V \times A$ in $[1]$.)

Group $\mathrm{C}^{\prime}$ consists of $\mathrm{C} 1, \mathrm{C} 2, \mathrm{C} 3$ and $\mathrm{C}^{\prime} 4$.

$C^{\prime} 4$. $\forall A \forall x \exists y \forall z(z \in y \mapsto z \in A \wedge z \in x)$.

$\mathrm{C}^{\prime} 4$ is Zermelo's Aussonderungsaxiom and is weaker than $\mathrm{C} 4$ (axiom of replacement). C4 follows, however, from $\mathrm{C}^{\prime} 4$ and other axioms.

Group F.

1. $7 \mathbb{S}(0)$.

2. $\mathbb{S}\left(\mathfrak{B}\left(H_{0}\right)\right)$, where $H_{0}$ is defined to be an abbreviation of $(K a)(\forall x \forall y(x \in a \wedge$ $(y \subseteq x \vee y \in x) \vdash y \in a) \wedge a \neq 0)$.

3. $\forall A \forall B(\&(A) \wedge A \subseteq B \vdash \mathbb{S}(B))$.

4. $\forall A \forall x(\mathbb{S}((A) \vdash \mathbb{S}(K\langle b a\rangle)(\langle b a\rangle \in A \wedge x \subseteq a \wedge x \in a)))$, where $(K\langle b a\rangle) \mathfrak{P}(b, a)$ is an abbreviation of $(K u) \exists b \exists a(u=\langle b a\rangle \wedge \mathfrak{H}(b, a))$. 
5. $\forall A(\mathbb{S}(A) \vdash(\mathbb{S}((K\langle b a\rangle)(a \times(\mathfrak{T}(A) \cap a) \in b)))$.

6. $\forall A\left(\forall x \leftrightarrow\left(A^{\prime \prime}\{x\}\right) \vdash(\Im)\left((K\langle b a\rangle) \forall x\left(x \in a \vdash\langle b a\rangle \in A^{\prime \prime}\{x\}\right)\right)\right)$.

Other than inferences in [1] we use infinite induction and \&-inference. In order to state $\$ S$-inference, we shall first define some notations. Let $U$ be a formula or a term and $a$ be a set variable not contained in $U$. Then we define $U^{a}$ from $U$ by transforming all the quantifiers and $\mathfrak{M}()$ in $U$ as follows: $\forall A, \exists B,(K x), \mathfrak{M}(T)$ to $\forall A(A \subseteq a \vdash), \exists B(B \subseteq a \wedge),(K x)(x \in a \wedge)$, $T^{a} \in a$ respectively.

Though deviating from [1], we define $a<b$ to be $a \cong b \wedge a \in b$. Then it is easily seen that $(\forall x \mathfrak{F}(x))^{a}$ is $\forall x\left(x<a \vdash \mathfrak{F}^{a}(x)\right)$.

$(b / \mathbb{B})$ (substitution of $b$ for $(\mathbb{B})$ is defined as follows. Let $U$ be a formula or a term. $U(b / \mathbb{S})$ is obtained from $U$ by transforming all the forms $\mathbb{E}(T)$ in $U$ to $T \in b$.

(\$3-inference. If $\mathfrak{A}$ is a clossed formula which holds, then

$$
\mathbb{S}\left((K\langle b a\rangle)\left(\mathfrak{A}^{a}(b /(\$))\right)\right) \text { also holds. }
$$

Infinite induction. If $\mathfrak{A}(i)$ holds for every actually given integer

$$
i(i=0,\{0\},\{0,\{0\}\}, \cdots) \text {, then } \forall i(i \in \omega \vdash \mathfrak{H}(i)) \text { holds. }
$$

REMARK. The following axiom $\mathrm{F}^{\prime}$ has a nicer form than $\mathrm{F} 5$ :

$\mathrm{F} 5^{\prime} . \quad \forall A(\$(A) \vdash \mathbb{S}((K\langle b a\rangle)(A \cap a \ni b)))$.

F5 follows form $\mathrm{F}^{\prime}$ (and other axioms) taking $V \times \mathfrak{D}(A)$ in place of $A$.

But the author is afraid if it is "too strong". It is to be noted that the axiom of the form

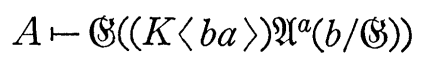

will lead to a contradiction by similar arguments as in [11] with the aid of 'Gödel number'. (See below.)

\section{$\S 2$. Some properties of the system.}

PROPOSITION 1. $\mathbb{B}(V)$.

Proof. This follows from $\mathrm{F} 2$ and F3.

Proposition 2.

$\forall x\left(x \in c \vdash \mathbb{S}\left(A^{\prime \prime}\{x\}\right)\right) \rightarrow \mathbb{S}\left(\left(K^{\prime}\langle b a\rangle\right) \forall x\left(x \in c \vdash\langle b a\rangle \in A^{\prime \prime}\{x\}\right)\right)$.

Proof. We define $B$ by the following:

$$
\begin{aligned}
& x \in c \rightarrow B^{\prime \prime}\{x\}=A^{\prime \prime}\{x\}, \\
& x \notin c \rightarrow B^{\prime \prime}\{x\}=V .
\end{aligned}
$$

We see clearly $\forall x\left(\$\left(B^{\prime \prime}\{x\}\right)\right.$. By F6 and F4 we have

$$
\mathbb{S}\left((K\langle b a\rangle)\left(\forall x\left(x \in a \vdash\langle b a\rangle \in B^{\prime \prime}\{x\}\right) \wedge c \subseteq a \wedge c \in a\right)\right),
$$


therefore, $\mathbb{\&}\left((K\langle b a\rangle)\left(\forall x\left(x \in c \vdash\langle b a\rangle \in B^{\prime \prime}\{x\}\right) \wedge c \subseteq a \wedge c \in a\right)\right)$. In virtue of this and F3 we have the proposition.

As a special case of Proposition 2 we have

Proposition 3. $\$(A), \mathbb{\$}(B) \rightarrow \$(A \cap B)$.

Definition. Let $\mathfrak{T}(A)$ be a term. $€(A, \mathfrak{I}, \alpha)$ is defined to be $(K a) \forall B\left(B^{\prime \prime}\{0\}=A \wedge \forall \beta\left(\beta<\alpha \vdash B^{\prime \prime}\{\beta+1\}=\mathfrak{I}(B\{\beta\})\right.\right.$

$\left.\wedge \forall \beta\left(\beta \leqq \alpha \wedge \beta \in K_{I I} \vdash B^{\prime \prime}\{\beta\}=(K x) \forall \gamma\left(\gamma<\beta \vdash x \in B^{\prime \prime}\{\gamma\}\right)\right) \vdash a \in B^{\prime \prime}\{\alpha\}\right)$.

The following proposition is familiar.

Proposition 4. $\subseteq(A, \mathfrak{T}, 0)=A$

$$
\mathfrak{C}(A, \mathfrak{I}, \alpha+1)=\mathfrak{I}(\mathfrak{\subseteq}(A, \mathfrak{T}, \alpha))
$$

$$
\alpha \in K_{I I} \rightarrow \mathfrak{S}(A, \mathfrak{I}, \alpha)=(K a) \forall \beta(\beta<\alpha \vdash a \in \mathfrak{C}(A, \mathfrak{I}, \beta)) .
$$

We have easily

Proposition 5. $\quad(\mathbb{S}(A), \forall X(\mathbb{S}(X) \vdash \mathscr{S}(\mathfrak{I}(X))) \rightarrow \forall \alpha(\mathbb{S}(\mathbb{S}(A, \mathfrak{T}, \alpha)))$.

Proposition 6. $\$(A), \forall X(\$(X) \vdash \mathbb{S}(\mathfrak{I}(X)))$

$$
\rightarrow \mathbb{B}((K\langle b a\rangle) \forall \alpha(\alpha \in a \vdash\langle b a\rangle \in \mathbb{S}(A, \mathfrak{B}, \alpha))) .
$$

Proof. We define $B$ as follows:

$$
\begin{aligned}
& B^{\prime \prime}\{x\}=\Subset(A, \mathfrak{I}, x) \text { if } x \in O n, \\
& B^{\prime \prime}\{x\}=V \text { otherwise. }
\end{aligned}
$$

Then we have the proposition by $\mathrm{F} 6$.

DEFINITION. $\mathbb{S}^{\circ}(A)$ is an abbreviation of $\mathbb{S}(\mathfrak{B}(A))$.

The following proposition is clear.

PROPOSITION 7.

1. $7 \mathbb{S}^{\circ}(0)$.

2. $\mathbb{S}^{\circ}\left(H_{0}\right)$.

3. $\forall A \forall B\left(\mathbb{S}^{\circ}(A) \wedge A \subseteq B \vdash \mathbb{S}^{\circ}(B)\right)$.

4. $\forall A \forall x\left(\mathbb{S}^{0}(A) \vdash \mathbb{S}^{0}((K a)(a \in A \wedge x \leqq a \wedge x \in a))\right)$.

5. $\forall A\left(\forall x\left(\mathbb{S}^{0}(A\{x\})\right) \rightarrow \mathbb{S}^{0}\left((K a) \forall x\left(x \in a \vdash a \in A^{\prime \prime}\{x\}\right)\right)\right.$.

6. $\forall x\left(x \in c \vdash \mathbb{S}^{0}\left(A^{\prime \prime}\{x\}\right)\right) \rightarrow \mathbb{S}^{\circ}\left((K a) \forall x\left(x \in c \vdash a \in A^{\prime \prime}\{x\}\right)\right)$.

7. $\mathbb{S}^{0}(A), \forall X\left(\mathbb{S}^{0}(X)-\mathbb{S}^{0}(\mathfrak{T}(X))\right) \rightarrow \mathbb{S}^{0}((K a) \forall \alpha(\alpha \in a-a \in \mathfrak{S}(A, \mathfrak{T}, \alpha)))$.

8. $\mathbb{S}^{0}(A), \forall X\left(\mathbb{S}^{0}(X) \vdash \mathbb{S}^{0}(\mathfrak{I}(X))\right) \rightarrow \forall \alpha\left(\mathbb{S}^{0}(\subseteq(A, \mathfrak{I}, \alpha))\right)$.

9. $\left.\mathbb{S}^{0}(A), \forall X\left(\mathbb{S}^{0}(X) \vdash \mathbb{S}^{0}((K a) \mathfrak{H}(a, X))\right) \rightarrow \mathbb{S}^{0}(K a) \forall \alpha(\alpha \in a \vdash \mathfrak{A}(\alpha, \mathfrak{S}(A, \mathfrak{I}, \alpha)))\right)$, where $\mathfrak{I}(X)$ is an abbreviation of $(K a) \mathfrak{A}(a, X)$.

Proposition 8. $\mathbb{S}^{\circ}(A), \mathfrak{F} \mathfrak{H c}(F) \rightarrow \mathbb{G}^{\circ}\left((K a)\left(a \in A \wedge \forall x\left(x \in a \vdash F^{\prime} x \in a\right)\right)\right)$.

Proof. We define $B$ by the following:

$$
\forall x\left(B^{\prime \prime}\{x\}=(K a)\left(a \in A \wedge F^{\prime} x \in a\right)\right) .
$$

By Proposition 7 we have $\forall x \mathbb{S}^{0}\left(B^{\prime \prime}\{x\}\right)$. Hence follows the proposition from Proposition 7.

Proposition 9. $\quad \mathbb{S}^{0}(A), \forall x\left(x \in a \vdash \mathfrak{F} \mathfrak{n c}\left(B^{\prime \prime}\{x\}\right)\right)$

$$
\rightarrow \mathbb{S}^{\circ}\left((K b)\left(b \in A \wedge \forall x \forall y\left(x \in a \wedge y \in b \vdash\left(B^{\prime \prime}\{x\}\right)^{\prime} y \in b\right)\right)\right) .
$$


PROOF. The proposition follows from Proposition 7 and Proposition 8.

We have axiom of replacement, that is,

Proposition 10. $\forall x \forall A\left(\mathfrak{u}_{\mathfrak{n}}(A) \vdash \exists y \forall u(u \in y \mapsto \exists v(v \in x \wedge\langle u v\rangle \in A))\right)$.

ProOF. In virtue of $C^{\prime} 4$, we have only to prove

$$
\forall x \forall A\left(\mathfrak{H} \mathfrak{i}(A) \vdash \exists y \forall v\left(v \in x \vdash A^{\prime} v \in y\right)\right),
$$

which is clear from Proposition 8.

Definition. $G_{0}$ is an abbreviation of $H_{0} \cap(K a) \mathfrak{P}^{a}$, where $\mathfrak{A}$ is the conjunction of the axiom A4 and axioms of Group C. Clearly we have $\mathbb{S}^{\circ}\left(G_{0}\right)$.

The following proposition is clear.

Proposition 11 .

1. $b \in a, c \in a \rightarrow(\{b c\})^{a}=\{b c\}$.

2. $a \in G_{0}, b \in a, c \in a \rightarrow(\langle b c\rangle)^{a}=\langle b c\rangle$.

3. $A \subseteq a, a \in G_{0} \rightarrow(\mathfrak{V}(A))^{a}=a \times A$,

where $a, b, c$, and $A$ are considered as variables.

Proposition 12. If $\mathfrak{B}(A)$ contains no \$s-symbol, $\forall X \mathfrak{B}(X)$ is closed and $\forall A\left(\mathbb{S}^{\circ}(A)-\mathfrak{B}(A)\right)$ holds, then $\forall A\left(\mathbb{S}^{0}(A) \vdash \mathbb{S}^{\circ}\left((K a) \mathfrak{B}^{a}(a \cap A)\right)\right)$ holds.

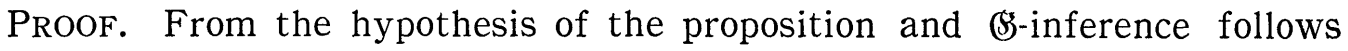
$\mathbb{S}(C)$, where $C$ is an abbreviation of

$$
(K\langle b a\rangle)\left(a \in G_{0} \wedge \forall x\left(x \cong a \wedge a \times x \in b \vdash \mathfrak{B}^{a}(x)\right)\right) .
$$

$B$ is defined to be

We have only to prove

$$
(K\langle b a\rangle)(a \times(A \cap a) \in b) .
$$

$$
\langle b a\rangle \in C, a \in A,\langle b a\rangle \in B \rightarrow \mathfrak{B}^{a}(a \cap A),
$$

which is clear.

DEFINITION. $M o(A)$ is an abbreviation of

$$
\forall F\left(\mathfrak{F n c}(F) \vdash \exists x\left(x \in A \wedge x \in H_{0} \wedge \forall y\left(y \in x \vdash F^{\prime} y \in x\right)\right)\right),
$$

which is called Mahlo's formula for $A$ and is essentially due to Mahlo ([5] and $[6])$.

$M(a, A)$ is an abbreviation of

$$
\forall x\left(x \subseteq a \wedge \mathfrak{F} \mathfrak{n c}(x) \vdash \exists y\left(y \in a \cap A \wedge y \in H_{0} \wedge \forall z\left(z \in y \vdash x^{\prime} z \in y\right)\right)\right) .
$$

The following proposition is clear.

PROPOSITION 13.

1. $a \in G_{0} \rightarrow(M o(A)) \mapsto M(a, A)$. (A is a variable.)

2. $7 M o(0)$.

3. $\forall A \forall x(M o(A) \vdash M o((K a)(a \in A \wedge x<a)))$.

4. $\forall A \forall B(M o(A) \wedge A \subseteq B \vdash M o(B))$.

$M o(A)$ has similar properties with $\mathbb{S}^{\circ}(A)$. 
Proposition 14. $M o(A)$, $\mathfrak{F} \mathfrak{c}(F) \rightarrow M o\left((K a)\left(a \in A \wedge \forall x\left(x \in a \vdash F^{\prime} x \in a\right)\right)\right)$.

Proof. $M o\left((K a)\left(a \in A \wedge \forall x\left(x \in a \vdash F^{\prime} x \in a\right)\right)\right)$ is equivalent to

$$
\begin{gathered}
\forall H\left(\mathfrak { \mho n c } ( H ) \vdash \exists y \left(y \in ( K a ) \left(a \in A \wedge \forall x\left(x \in a \vdash F^{\prime} x \in a\right)\right.\right.\right. \\
\left.\left.\left.\wedge y \in H_{0} \wedge \forall z\left(z \in y \vdash H^{\prime} z \in y\right)\right)\right)\right) .
\end{gathered}
$$

We have, therefore, only to prove

$$
\begin{aligned}
& M o(A), \mathfrak{F} \mathfrak{m c}(F), \mathfrak{F} \mathfrak{m c}(H) \rightarrow \exists y\left(y \in A \wedge y \in H_{0}\right. \\
& \left.\wedge \forall x\left(x \in y \vdash F^{\prime} x \in y\right) \wedge \forall z\left(z \in y \vdash H^{\prime} z \in y\right)\right),
\end{aligned}
$$

which is clear by introducing new function $F_{1}$ as follows:

$$
F_{1}^{\prime} x=\left\{F^{\prime} x, H^{\prime} x\right\} .
$$

Moreover, we have the following proposition.

PROPOSITION 15. $\quad \mathbb{S}^{\circ}(A) \rightarrow M o(A)$.

PROOF. The proposition is clear by Proposition 8.

However, the following example shows the difference between $\$^{\circ}(A)$ and $M o(A)$. We set

$$
A=G_{0}
$$

and

$$
B=(K a) \exists x\left(\mathfrak{F n c}(x) \wedge \forall i \forall j\left(i \in \omega \wedge j \in \omega \wedge i<j \vdash \overline{\overline{x^{\prime}} i}<\overline{\overline{x^{\prime} j}}\right) \wedge a=\bigcup_{i \in \omega} x^{\prime} i\right) .
$$

Then we see $M o(A), M o(B)$ and $A \cap B=0$.

Proposition 16. $\quad \$^{\circ}(A) \rightarrow \$^{\circ}((K a) M(a, A))$.

Proof. This follows from Proposition 12 and 13.

Proposition 17. $\mathbb{S}^{0}(A) \rightarrow \mathbb{S}^{0}((K a) \forall \alpha(\alpha \in a \vdash M(a, \mathfrak{S}(A, \mathfrak{I}, \alpha))))$, where $\mathfrak{I}(X)$ is an abbreviation of $(K a) M(a, X)$.

Proof. This follows from Propositions 15 and 7.

A term $T\left(A_{1}, \cdots, A_{n}\right)$ is called elementary, if it is contructed just by $E$, $\cap,-, \mathfrak{D}, \mathfrak{B},\left(\mathfrak{S} \mathfrak{n}, \boldsymbol{S} \mathfrak{n}_{2}, \boldsymbol{S} \mathfrak{n}_{3}, A_{1}, \cdots, A_{n}\right.$.

If a term $T$ is elementary and closed, then we define Gödel number $g(T)$ of $T$ as follows:

1. $g(E)=3$.

2. $g\left(T_{1} \cap T_{2}\right)=2 \cdot 3^{g\left(T_{1}\right)} \cdot 5^{g\left(T_{3}\right)}$.

3. $g(-T)=2^{2} \cdot 3^{g(T)}$.

4. $g(\mathfrak{D}(T))=2^{3} \cdot 3^{g(T)}$.

5. $g(\mathfrak{B}(T))=2^{4} \cdot 3^{g(T)}$.

6. $g(\mathbb{C n n}(T))=2^{5} \cdot 3^{g(T)}$.

7. $g\left(\mathbb{S} \mathfrak{n v}_{2}(T)\right)=2^{6} \cdot 3^{g(T)}$.

8. $g\left(\Re_{\left.\mathfrak{n} \mathfrak{v}_{3}(T)\right)}=2^{7} \cdot 3^{g(T)}\right.$.

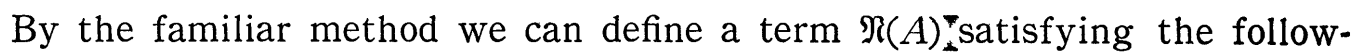
ing conditions : 
1. $\Re(3)=E$.

2. $a \in \omega, b \in \omega \rightarrow \Re\left(2 \cdot 3^{a} \cdot 5^{b}\right)=\Re(a) \cap \Re(b)$.

3. $a \in \omega \rightarrow \Re\left(2^{2} \cdot 3^{a}\right)=-\Re(a)$.

4. $a \in \omega \rightarrow \Re\left(2^{3} \cdot 3^{a}\right)=\mathfrak{I}(\mathfrak{N}(a))$.

5. $a \in \omega \rightarrow \mathfrak{N}\left(2^{4} \cdot 3^{a}\right)=\mathfrak{B}(\mathfrak{N}(a))$.

6. $a \in \omega \rightarrow \Re\left(2^{5} \cdot 3^{a}\right)=\mathfrak{N n}(\Re(a))$.

7. $a \in \omega \rightarrow \mathfrak{N}\left(2^{6} \cdot 3^{a}\right)=\left(\mathfrak{S n v}_{2}(\mathfrak{N}(a))\right.$.

8. $\quad a \in \omega \rightarrow \Re\left(2^{7} \cdot 3^{a}\right)=\left(\mathfrak{n n}_{3}(\Re(a))\right.$.

According to $[\mathbf{1}]$, a sequence of natural numbers $n_{0}, n_{1}, n_{2}, \cdots$ are called "definable (in this system)" if and only if there exists a term $T(i)$ satisfying the following condition:

1. $T(i)=n_{i}$ for every $i(i=0,1,2, \cdots)$.

2. $T(0)$ has no free variable.

A sequence $\mathfrak{A}_{0}, \mathfrak{2}_{1}, \mathfrak{A}_{2}, \cdots$ of closed formulas is called "definable" if and only if the sequence of usual Gödel numbers of $\mathfrak{A}_{0}, \mathfrak{A}_{1}, \mathfrak{A}_{2}, \cdots$ (not in the sense $g(T))$ is definable.

We see clearly that if the sequence $\mathfrak{A}_{0}, \mathfrak{A}_{1}, \mathfrak{A}_{2}, \cdots$ is definable, then the sequence

is also definable.

$$
g\left((K\langle b a\rangle)\left(\mathfrak{R}_{i}^{a}(b / \mathfrak{S}) \wedge a \in G_{0}\right)\right) \quad(i=0,1,2, \cdots)
$$

Let $\mathfrak{A}_{0}, \mathfrak{A}_{1}, \mathfrak{A}_{2}, \cdots$ be a definable sequence of provable formulas. Then there exists a term $T(i)$ such that

$$
T(i)=g\left((K\langle b a\rangle)\left(\mathfrak{H}_{i}^{a}(b / \mathbb{S}) \wedge a \in G_{0}\right)\right) \quad(i=0,1,2, \cdots) .
$$

Then $\forall i(i \in \omega \vdash \mathfrak{S}(\mathfrak{N}(T(i))))$. Therefore we have

$$
\leftrightarrow((K\langle b a\rangle) \forall i(i \in \omega \vdash\langle b a\rangle \in \Re(T(i)))),
$$

which we set as $\$(A)$. Here $A$ means the class of all the super-complete inner models, in which $\mathfrak{A}_{0}, \mathfrak{A}_{1}, \mathfrak{A}_{2}, \cdots$ and Bernays-Gödel's set theory hold.

Let $\mathfrak{A}_{0}, \mathfrak{A}_{1}, \mathfrak{A}_{2}, \cdots$ be a definable sequence of closed provable formulas and $\mathfrak{B}_{0}, \mathfrak{B}_{1}, \mathfrak{B}_{2}, \cdots$ be a definable sequence of all the axioms (which contains axioms of equality other than $A, B^{\prime}, C^{\prime}, D, E$ and $F$ ). Then we define $\mathbb{s}_{0}, \mathbb{s}_{1}, \mathbb{s}_{2}, \cdots$ as follows :

$$
\begin{aligned}
& \mathfrak{E}_{0} \text { is } \mathfrak{A}_{0} \wedge \mathfrak{B}_{0} . \\
& \mathfrak{E}_{2 n+1} \text { is } \mathbb{S}\left((K\langle b a\rangle)\left(\mathfrak{E}_{2 n}^{a}(b / \mathfrak{G}) \wedge a \in G_{0}\right)\right) \quad(n=0,1,2, \cdots) . \\
& \mathfrak{S}_{2 n+2} \text { is } \quad \mathfrak{S}_{0} \wedge \cdots \wedge \mathfrak{E}_{2 n+1} \wedge \mathfrak{A}_{n+1} \wedge \mathfrak{B}_{n+1} \quad(n=0,1,2, \cdots) .
\end{aligned}
$$

The sequence $\mathfrak{E}_{0}, \mathfrak{E}_{1}, \mathfrak{E}_{2}, \cdots$ is clearly definable and is called a derived sequence of $\mathfrak{A}_{0}, \mathfrak{A}_{1}, \mathfrak{A}_{2}, \cdots$.

The following proposition is proved by the familiar method.

Proposition 18. If $\mathfrak{A} \rightarrow \mathfrak{B}$ is closed and is probable without infinite induc- 
tion or B-inference or axioms $\mathrm{A}, \mathrm{B}^{\prime}, \mathrm{C}^{\prime}, \mathrm{D}, \mathrm{E}, \mathrm{F}$, then also

$$
\exists x(x<a), \mathfrak{P}^{a}(b / \mathbb{B}) \rightarrow \mathfrak{B}^{a}(b / \mathbb{S})
$$

is provable without infinite induction or $\$\left(\right.$-inferece or axioms $\mathrm{A}, \mathrm{B}^{\prime}, \mathrm{C}^{\prime}, \mathrm{D}, \mathrm{E}, \mathrm{F}$.

PROPOSITION 19. Let $\mathfrak{A}_{0}, \mathfrak{A}_{1}, \mathfrak{R}_{2}, \cdots$ be a definable sequence of closed probable formulas and $\mathfrak{S}_{0}, \mathfrak{S}_{1}, \mathfrak{S}_{2}, \cdots$ be a derived sequence of $\mathfrak{A}_{0}, \mathfrak{A}_{1}, \mathfrak{H}_{2}, \cdots$. If $\mathfrak{B}$ is prova-

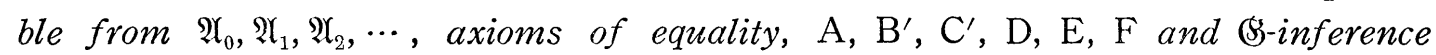
(but without infinite induction), then there exists $\mathfrak{B}_{n}$ such that $\mathfrak{C}_{n} \rightarrow \mathfrak{B}$ is provable without infinite induction or \$B-inference, or A, B', C', D, E, F.

PROOF. We prove this by induction on the number of inferences to prove $\mathfrak{B}$. Clearly we have only to prove this in the case where $\mathfrak{B}$ is of the form

$$
\mathbb{S}\left((K\langle b a\rangle) \mathbb{S}^{a}(b / \mathbb{S})\right)
$$

and there exists $\mathfrak{E}_{m}$ such that $\mathfrak{\mho}_{m} \rightarrow \mathfrak{D}$ is provable without infinite induction or \$s-inference, or A, B', C', D, E, F. By Proposition 18 we see easily that

$$
(K\langle b a\rangle) \mathfrak{D}^{a}(b / \mathbb{S}) \supseteqq(K\langle b a\rangle)\left(\left(_{m}^{a}(b / \mathbb{S}) \wedge a \in G_{0}\right)\right.
$$

is provable without infinite induction or $\$$-inference or $A, B^{\prime}, C^{\prime}, D, E, F$. Hence follows the proposition.

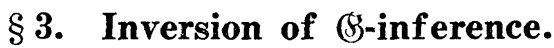

In this section we shall consider the following inference as a basic inference :

Inversion of $\left(\mathfrak{S}\right.$-inference. If $\mathfrak{X}$ is a closed formula and $\mathbb{S}\left((K\langle b a\rangle)\left(\mathfrak{H}^{a}(b / \mathbb{S})\right)\right)$ holds, then $\mathfrak{A}$ also holds.

First we shall reduce axioms $C^{\prime} 4, D$ and $E$ to the following weaker axioms $\mathrm{C} 4^{\prime}, \mathrm{D}^{\prime}$ and $\mathrm{E}^{\prime}$.

C4'. $\forall x \forall y \exists z \forall u(u \in z \mapsto u \in x \wedge u \in y)$.

$\mathrm{D}^{\prime} . \quad \forall x(7 \operatorname{sm}(x)-\exists y(y \in x \wedge(\mathfrak{r} r(y, x)))$.

E'. $\forall x(\forall u(u \in x \vdash u \neq 0) \wedge \forall u \forall v(u \in x \wedge v \in x \wedge u \neq v \vdash(\mathfrak{r}(u, v))$

$$
\vdash \exists y \forall z(z \in x \vdash \exists ! u(u \in z \cap y))) \text {. }
$$

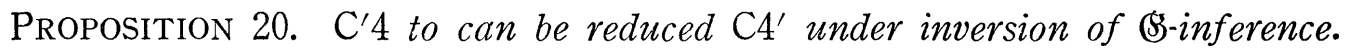

Proof. We have only to prove $\mathbb{S}^{\circ}(B)$, where $B$ is

$$
(K a) \forall z \forall x(z \subseteq a \wedge x<a \vdash \exists y(y<a \wedge \forall u(u<a \vdash(u \in y \mapsto u \in x \wedge u \in z)))) .
$$

Clearly $H_{0} \subseteq B$ under $\mathrm{C}^{\prime}$ therefore we have the proposition.

PROPOSITION 21. D can be reduced to $\mathrm{D}^{\prime}$ under inversion of $\$ \$$-inference.

Proof. We have only to prove $\mathscr{S}^{\circ}(B)$, where $B$ is

$$
(K a) \forall z \forall x(z \subseteq a \wedge x<a \wedge x \in z \vdash \exists y(y<a \wedge y \in z \wedge \forall u(u<a \vdash 7(u \in z \wedge u \in y)))) .
$$

Clearly $H_{0} \cong B$ follows from $\mathrm{D}^{\prime}$, therefore we have the proposition. 
Proposition 22. Axioms $E$ can be reduced to $E^{\prime}$ under inversion of $\mathscr{B}$ inference.

Now we shall reduce Group $B^{\prime}$ to Group $B$. To the end, we use the method of $[\mathbf{1 0}]$. As we use Gentzen's sequence in $[\mathbf{1 0}]$, we shall explain notations. We say 'a closed formula $\mathfrak{P}$ is an axiom of a system $S$ ', if and only if ' $\rightarrow \mathfrak{A}$ is a beginning sequence of $S$ '.

The system $S_{0}$ is obtained from our original system by adding the inversion of $\$$-inference.

Group $B^{\prime \prime}$ is defied by

$$
\forall X_{1} \cdots \forall X_{m} \forall x_{1} \cdots \forall x_{n} \exists Y \forall x(x \in Y \mapsto \mathfrak{A}(x)) .
$$

$S_{1}$ is obtained from $S$ by replacing Group $B^{\prime}$ by Group B" and all the axioms and inferences containing $K$ other than Group $\mathrm{B}^{\prime}$ by an equivalent axiom without $K$, e.g.

is replaced by

$$
\mathfrak{S}((K\langle b a\rangle) \mathfrak{A} a(b / \mathfrak{S}))
$$

$$
\forall X\left(\forall u\left(u \in X \mapsto \exists b \exists a\left(u=\langle b a\rangle \wedge \mathfrak{A}^{a}(b /(\mathbb{S}))\right) \vdash \mathbb{\$}(X)\right),\right.
$$

which we shall denote by $\rho(\mathfrak{H})$.

Though our system contains infinite induction, the method of [10] is still valid for our system. $((K u) \mathfrak{A}(u))^{*}$ is defined as $\{X\} \forall x\left(x \in X \mapsto \mathfrak{H}^{*}(x)\right)$, while other *-operations are defined similarly as in [10]. Then we have the following

Lemma. Let $\mathfrak{X}$ be a formula and $T$ a term with only free variable $A_{1}, \cdots, A_{n}$. Then the following sequences are provable simultaneously in $S_{1}$ :

$$
\begin{aligned}
& a \in H_{0}, A_{1} \leqq a, \cdots, A_{n} \leqq a \rightarrow \mathfrak{I}^{* a}(b / \mathfrak{S}) \mapsto\left(\mathfrak{P}^{a}(b / \mathbb{S})\right)^{*} ; \\
& a \in H_{0}, A_{1} \leqq a, \cdots, A_{n} \leqq a \rightarrow A \leqq a \wedge T^{* a}(b / \mathbb{S})(A) \mapsto\left(T^{a}(b /(\mathfrak{S}))^{*}(A) .\right.
\end{aligned}
$$

Propositions $1-5$ of $[\mathbf{1 0}]$ can be proved for our system by the literally same proof and Proposition 6 of $[\mathbf{1 0}]$ can be proved for our system as follows:

Proposition 6 of [10], If $\Gamma \rightarrow \Delta$ is provable in $S_{0}$, then $\Gamma^{*} \rightarrow \Delta^{*}$ is provable in $S_{1}$.

Proof. Suppose $\Gamma \rightarrow \Delta$ be a beginning sequence. Then the proposition is clear. Therefore we have only to prove the proposition under the hypothesis that $\Pi^{*} \rightarrow \Lambda^{*}$ is provable in $S_{1}$ for every upper sequence $\Pi \rightarrow \Lambda$ of $\Gamma \rightarrow \Delta$. We have only to consider the following cases:

$$
\rightarrow \underset{\mathfrak{A}}{\left.\rightarrow \mathbb{S}((K<b a\rangle) \mathfrak{A}^{a}(b / \mathfrak{S})\right)}
$$

where $\Gamma \rightarrow \Delta$ is $\rightarrow\left(\mathbb{S}\left((K\langle b a\rangle) \mathfrak{A}^{a}(b / \mathbb{S})\right)\right.$. By the hypothesis we have $\rightarrow \mathfrak{H}^{*}$ therefore we have

$$
\rightarrow \forall X(\forall u(u \in X \mapsto \exists b \exists a(u=\langle b a>\wedge \mathfrak{A} * a(b / \mathbb{S}))) \vdash \mathbb{S}(X)),
$$


whence follows the proposition by the preceding Lemma.

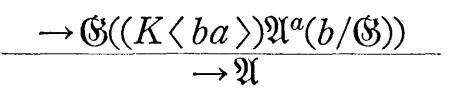

where $\Gamma \rightarrow \Delta$ is $\rightarrow \mathfrak{A}$. This case is proved by the same way as in 1 ).

$$
\underset{\rightarrow \mathfrak{A}(0) \rightarrow \mathfrak{A}(1) \rightarrow \mathfrak{A}(2) \cdots}{\rightarrow \forall x(x \in \omega \vdash \mathfrak{A}(x))}
$$

where $\Gamma \rightarrow \Delta$ is $\rightarrow \forall x(x \in \omega \vdash \mathfrak{A}(x))$. By the hypothesis the following sequences are provable in $S_{1}$ :

$$
\rightarrow \mathfrak{A} *(0), \quad \rightarrow \mathfrak{A} *(1), \quad \rightarrow \mathfrak{A}^{*}(2), \cdots,
$$

therefore we have $\rightarrow \forall x(x \in \omega \vdash \mathfrak{A}(x))$, whence follows $\rightarrow(\forall x(x \in \omega \vdash \mathfrak{P}(x)))^{*}$.

Therefore we can reduce $S_{0}$ to $S_{1}$. Now we shall prove that Group B" can be reduced to Group B. To the end, we have only to prove $\forall u(u \in A \mapsto \mathfrak{A}(u))$ $\vdash \mathbb{S}(A)$, where $\mathfrak{A}(u)$ is

$$
\begin{gathered}
\exists b \exists a\left(u=\langle b a\rangle \wedge \forall z_{1} \cdots \forall z_{m} \forall x_{1} \cdots \forall x_{n}\left(z_{1} \subseteq \alpha \wedge \cdots \wedge z_{n} \leqq a \wedge x_{1}<a \wedge \cdots \wedge x_{n}<a\right.\right. \\
\vdash \exists y(y \subseteq a \wedge \forall x(x<a \vdash(x \in y \mapsto B(x))))))
\end{gathered}
$$

and $\mathfrak{B}$ does not contain $\mathfrak{S}$. Clearly we have only to prove $\mathfrak{B}\left(H_{0}\right) \subseteq A$, which follows from Group B and the other axioms (mainly $\mathrm{C}^{\prime} 4$ ).

Now, we shall reduce the inversion of $\$ s$-inference. $S_{2}$ is obtained from $S_{1}$ by excluding inversion of \$s-inference.

First we shall prove the following lemma.

LEMMA. Let $\Delta_{0}$ be a system of axioms and consistent with $S_{2}$. If $\rho(\mathfrak{R})$ is provable from $\Delta_{0}$ is $S_{2}$, then $\mathfrak{A}$ and $\Delta_{0}$ are consistent from $\mathfrak{A}$ and $\Delta_{0}$ are consistent with $S_{2}$.

Proof. We have only to prove that if $\Gamma \rightarrow \Delta$ is provable from $\mathfrak{\imath}$ and $\Delta_{0}$. in $S_{2}$, then $\mathfrak{A}, \Gamma \rightarrow \Delta$ is provable from $\Delta_{0}$ in $S_{2}$.

If $\Gamma \rightarrow \Delta$ is $\rightarrow \mathfrak{A}$ or $\rightarrow \mathfrak{B}$, where $\mathfrak{B}$ is an axiom of $\Delta_{0}$, or a beginning sequence of $S_{2}$, then $\mathfrak{A}, \Gamma \rightarrow \Delta$ is clearly provable from $\Delta_{0}$ in $S_{2}$.

Therefore we have only to prove that $\mathfrak{A}, \Gamma \rightarrow \Delta$ is provable from $\Delta_{0}$ in $S_{2}$ under the hypothesis that $\mathfrak{A}, \Pi \rightarrow \Lambda$ is provable from $\Delta_{0}$ in $S_{2}$ for every upper sequence $\Pi \rightarrow \Lambda$ of $\Gamma \rightarrow \Delta$.

We have only to prove the case where $\Pi \rightarrow \Lambda$ is $\rightarrow \mathfrak{B}$ and $\Gamma \rightarrow \Delta$ is $\rightarrow \rho(\mathfrak{B})$.

From the hypothesis $\rightarrow \rho(\mathfrak{A} \vdash \mathfrak{B})$ and $\rightarrow \rho(\mathfrak{H})$ are provable from $\Delta_{0}$ in $S_{2}$, therefore $\rightarrow \rho(\mathfrak{B})$ is also provable from $\Delta_{0}$ in $S_{2}$.

In virtue of this lemma we can easily see that the inversion of $\$$-inference holds in the maximal consistent system containing $S_{2}$, which we can obtain by the usual method.

Proposition 23. If $\mathrm{A}, \mathrm{B}, \mathrm{C}^{\prime}, \mathrm{D}^{\prime}, \mathrm{F}$, Bs-inference and infinite induction are

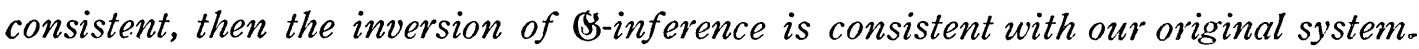




\section{$\S 4$. Another system of axioms.}

In this section we shall give another system of set theory with strong axioms of infinity, which is closely related to the former system.

The new system is obtained from the former system by the following modifications :

1) Introduce new basic symbols $\Im()$ and $\Im($,$) and eliminate \$$

2) Replace Group $\mathrm{F}$ and $\$$-inference by the following Group $\mathrm{H}$ and $\Im$-inference. Group $\mathrm{H}$.

1. $7 \Im(0)$.

2. $\Im\left(H_{0}\right)$, where $H_{0}$ is defined to be an abbreviation of

$(K a)(\forall x \forall y(x \in a \wedge(y \in x \vee y \leqq x) \vdash y \in a) \wedge a \neq 0)$.

3. $\forall A \forall B(\Im(A) \wedge A \subseteq B \vdash \Im(B))$.

4. $\forall A \forall x(\Im(A) \vdash \Im((K a)(a \in A \wedge x<a)))$, where $b<a$ is an abbreviation of $b \subseteq a \wedge b \in a$.

5. $\forall A\left(\forall x \Im\left(A^{\prime \prime}\{x\}\right) \vdash \Im\left((K a) \forall x\left(x \in a \vdash a \in A^{\prime \prime}\{x\}\right)\right)\right)$.

6. $\forall x \forall A(\Im(x, A) \mapsto \Im(x, x \cap A))$.

7. $\forall A(\Im(A) \vdash \Im((K a) \Im(a, A)))$.

$\Im$-inference.

If $\mathfrak{A}$ is a closed formula and holds, then $\mathfrak{\Im}\left(K(a) \mathfrak{P}^{a}\right)$ also holds.

The following properties are also proved in the new system in the same way as in the former system.

PROPOSITION 1. $\Im(V)$.

PROPOSITION 2. $\forall x\left(x \in c \vdash \Im\left(A^{\prime \prime}\{x\}\right)\right) \rightarrow \Im\left((K a) \forall x\left(x \in c \vdash a \in A^{\prime \prime}\{x\}\right)\right)$.

PROPOSITION 3. $\Im(A), \mathfrak{J}(B) \rightarrow \Im(A \cap B)$.

Defnition. Let $\mathfrak{T}(A)$ be a term. $\subseteq(A, \mathfrak{I}, \alpha)$ is defined to be $(K a) \forall B\left(B^{\prime \prime}\{0\}=A \wedge \forall \beta\left(\beta<\alpha \vdash B^{\prime \prime}\{\beta+1\}=\mathfrak{I}\left(B^{\prime \prime}\{\beta\}\right)\right)\right.$ $\left.\wedge \forall \beta\left(\beta \leqq \alpha \wedge \beta \in K_{I I} \vdash B^{\prime \prime}\{\beta\}=(K x) \forall \gamma\left(\gamma<\beta \vdash x \in B^{\prime \prime}\{\gamma\}\right)\right) \vdash a \in B^{\prime \prime}\{\alpha\}\right)$.

PROPOSITION 4. $\mathfrak{S}(A), \forall X(\mathfrak{S}(X) \vdash \mathfrak{I}(\mathfrak{I}(X))) \rightarrow \mathfrak{I}(K a) \forall \alpha(\alpha \in a \vdash a \in \mathfrak{S}(A, \mathfrak{I}, \alpha))$.

Proposition 5. $\Im(A), \mathfrak{\mho n c}(F) \rightarrow \Im\left((K a)\left(a \in A \wedge \forall x\left(x \in a \vdash F^{\prime} x \in a\right)\right)\right)$.

Proposition 6. $\Im(A), \forall x\left(x \in a \vdash \mathfrak{F n c}\left(B^{\prime \prime}\{x\}\right)\right)$

$$
\rightarrow \mathfrak{J}\left((K b)\left(b \in A \wedge \forall x \forall y\left(x \in a \wedge y \in b \vdash\left(B^{\prime \prime}\{x\}\right)^{\prime} y \in b\right)\right)\right) .
$$

Proposition 7. $\forall x \forall A(\mathfrak{U} \mathfrak{n}(A) \vdash \exists y \forall u(u \in y \mapsto \exists v(v \in x \wedge\langle u v\rangle \in A)))$.

Proposition 8. If $\mathfrak{B}(A)$ contains no free variable other than $A$ and $\forall A(\Im(A) \vdash B(A))$ holds, then $\forall A\left(\Im(A) \vdash \Im\left((K a) \mathfrak{B}^{a}(a \cap A)\right)\right)$ also holds.

PROOF. In virtue of $\Im$-inference follows $\Im(C)$, where $C$ is an abbreviation of

$$
(K a) \forall x(x \subseteq a \wedge \Im(a, x) \vdash \mathfrak{B}(x)) .
$$

$\mathbb{B}$ is defined to be $(K a) \mathfrak{S}(a, A)$. We have only to prove

$$
a \in C, a \in B \rightarrow \mathfrak{B}^{a}(a \cap A),
$$


which is clear.

PROPOSITION 9. $\Im(A) \rightarrow M o(A)$.

Proposition 10. $\Im(A) \rightarrow \Im((K a) M(\alpha, A))$.

PROPOSITION 11. $\Im(A) \rightarrow \mathfrak{I}((K a) \forall \alpha(\alpha \in a \vdash M(a, \subseteq(A, \mathfrak{I}, \alpha))))$, where $\mathfrak{I}(X)$ is an abbreviation of $(K a) M(a, X)$.

$I_{n}$ is defined to be

$$
(K a) \exists x \exists y(a=\langle x y\rangle \wedge \mathfrak{J}(x, y)) .
$$

A term $T\left(A_{1}, \cdots, A_{n}\right)$ is called elementary, if it is constructed just by $E$,

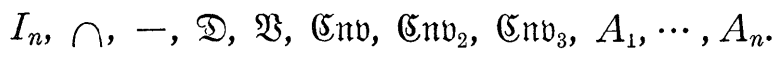

If a term $T$ is elementary and closed, then we define Gödel number $g(T)$, of $T$ in the same way as in $\S 2$, adding the condition

$$
g\left(I_{n}\right)=7 \text {. }
$$

We define a term $\mathfrak{N}(A)$ in the same way as in $\S 2$, adding the condition

$$
\mathfrak{R}(7)=I_{n} .
$$

The concepts that a sequence of natural numbers and a sequence of closed formulas are 'definable' are defined in the same way as in $\S 2$.

Then for any definable sequence $\mathfrak{A}_{0}, \mathfrak{A}_{1}, \mathfrak{A}_{2}, \cdots$ of provable formulas, there exists a term $T(i)$ such that

$$
T(i)=g\left((K a)\left(\mathfrak{R}_{i}^{a} \wedge a \in G_{0}\right)\right) .
$$

By an analogous argument as in $\S 2$, we have

$$
\Im((K a) \forall i(i \in \omega \vdash a \in \mathfrak{R}(T(i)))),
$$

which we set as $\Im(A)$. Here $A$ means the class of all the complete inner models, in which $\mathfrak{A}_{0}, \mathfrak{A}_{1}, \mathfrak{A}_{2}, \cdots$ and Bernays-Gödel's set theory hold.

A derived sequence of a definable sequence of closed provable formulas is defined in the same way as in $\S 2$, by replacing the axiom $F$ there by the axiom $\mathrm{H}$.

Proposition 12. Let $\mathfrak{H}_{0}, \mathfrak{A}_{1}, \mathfrak{A}_{2}, \cdots$ be a definable sequence of closed provable formulas and $\mathfrak{E}_{0}, \mathfrak{E}_{1}, \mathfrak{E}_{2}, \cdots$ be a derived sequence of $\mathfrak{H}_{0}, \mathfrak{A}_{1}, \mathfrak{H}_{2}, \cdots$. If $\mathfrak{B}$ is provable from $\mathfrak{H}_{0}, \mathfrak{A}_{1}, \mathfrak{A}_{2}, \cdots$, axiom of equality, $\mathrm{A}, \mathrm{B}^{\prime}, \mathrm{C}^{\prime}, \mathrm{D}, \mathrm{E}, \mathrm{H}$, and $\mathfrak{\Im}_{\text {-inference (but }}$ without infinite induction), then there exists $\mathfrak{S}_{n}$ such that $\mathfrak{S}_{n} \rightarrow \mathfrak{B}$ is provable without infinite induction or J-infinite or $\mathrm{A}, \mathrm{B}^{\prime}, \mathrm{C}^{\prime}, \mathrm{D}, \mathrm{E}, \mathrm{H}$.

We shall further consider the following inference as a basic inference.

Inversion of $\Im$-inference.

If $\mathfrak{A}$ is a closed formula and $\mathfrak{S}\left((K a) \mathfrak{P}^{a}\right)$ holds, then $\mathfrak{A}$ also holds.

PROPOSITION 13. Under inversion of $\Im$-inference, $\mathrm{C}^{\prime} 4, \mathrm{D}$ and $\mathrm{E}$ can be reduced to $\mathrm{C}^{\prime} 4, \mathrm{D}^{\prime}$ and $\mathrm{E}^{\prime}$, respectively.

Group B'" is defined to be all the axioms of Group B and the following B9. 
B9. $\exists A \forall x \forall y(\langle x y\rangle \in A \mapsto \Im(x, y))$.

Proposition 14. If $\mathrm{A}, \mathrm{B}^{\prime \prime \prime}, \mathrm{C}^{\prime}, \mathrm{D}^{\prime}, \mathrm{H}$, ऽ-inference and infinite induction are consistent, then the inversion of $\mathfrak{\Im}$-inference are consistent with our original system.

Institute for Advanced Study, Tokyo University of Education

\section{References}

[1] K. Gödel, The consistency of the axiom of choice and of the gendralized continuum-hypothesis with the axioms of set theory, Revised ed., Princeton, 1951.

[2] K. Gödel, Über formal unentscheidbare Sätze der Principia Mathematica und verwandter System I, Monatsh. Math. Ph., 38 (1930), 173-198.

[3] A. Lévy, Contributions to the metamathematics of set theory, (Hebrew with English summary) 1958, Thesis, Hebrew University, Jerusalem.

[4] A. Lévy, Axiom schemata of strong infinity in axiomatic set theory, Pacific J. Math., 10 (1960), 223-238.

[ 5 ] P. Mahlo, Über lineare transfinite Mengen, Ber. Leipzig, 63 (1911), 187-225.

[6] P. Mahlo, Zur Theorie und Anwendung der $\rho_{0}$-Zahlen, Ibid., 64 (1912), 108-112; 65 (1913), 268-282.

[7] J. B. Rosser, Extensions of some theorems of Gödel und Church, J. S. L., 1 (1936) $87-91$.

[ 8 ] J. C. Shepherdson, Inner models for set theory, J.S. L., (1951), 161-190; (1952), 225-237.

[9] K. Schütte, Beweistheoritische Erfassung der unendlichen Induktion in der Zahlen Theorie, Math. Ann., 122 (1951). 369-389.

[10] G. Takeuti, A metamathematical theorem on functions, J. Math. Soc. Japan, 8 (1956), 65-78.

[11] G. Takeuti, Remark on the truth definition, J. Math. Soc. Japan, 13 (1961), 207209.

[12] A. Tarski, Der Wahrheitsbegriff in den formalisierten Sprachen, Studia Philos., 1 (1936), 26-405.

[13] A. Tarski, Über unerreichbare Kardinalzahlen, Fund. Math., 30 (1938), 68-89.

[14] A. Tarski, A. Mostowski and R. M. Robinson, Undecidable Theories, Amsterdam, 1953. 